\title{
Les Simuliidae (Diptera, Nematocera) de la Neste d'Aure (Hautes-Pyrénées) II. Ecologie et impact des aménagements dans la moyenne et basse vallée
}

\author{
M. Clergue-Gazeau 1 \\ D. Holmière ${ }^{1}$ \\ S. Meurgues 1 \\ E. Angelier 1
}

Mots clés: Ecologie, eaux courantes, Simuliidae, aménagements, Pyrénées.

Une étude -dérive et benthos. de la basse et moyenne Neste d'Aure (Hautes-Pyrénées) a permis de recenser 26 espèces de Diptères Simuliidae. L'analyse factorielle des correspondances met en évidence deux associations successives entre $1100 \mathrm{~m}$ et $427 \mathrm{~m}$, en fonction de la pente et des temperatures estivales. La classification par la méthode des nuées dynamiques affine ces résultats en soulignant l'effet des aménagements hydro-électriques (biefs à débit réservé) sur le peuplement.

Simulidae (Diptera, Nematocera) from the Neste d"Aure (Central Pyrenees) II. Ecology and effect of water management on the middle and lower valley.

Keywords : Ecology, running waters, Simuliidae, water management, Pyrenees.

A study of drift and benthos in the lower and middle reaches of the river Neste d'Aure produced 26 species of Simuliidae. Factorial analysis indicated two successive associations between altitudes of $1100 \mathrm{~m}$ and $427 \mathrm{~m}$, and these associations were a function of slope and summer temperatures. The classification produced by cluster analysis improved these results by emphasizing the effect of hydro-electric management (level for reserve flow) on the population.

Le but de cette étude est de compléter nos connaissances sur les Simuliidae de la Neste d'Aure (Hautes-Pyrénées), telles qu'elles résultent des recherches de Lavandier (1976) et Clergue-Gazeau \& Gazagnes (1986). Nos propres recherches ont porté sur la moyenne et basse vallée de la Neste, de 1060 m au confluent avec la Garonne, à $427 \mathrm{~m}$ d'altitude, sur une longueur de $52 \mathrm{~km}$.

\section{La Neste d'Aure (fig. 1)}

Elle est caractérisée par une orientation ouest-est du cours supérieur, sud-nord du cours moyen, et à

\footnotetext{
1. Laboratoire d'Hydrobiologie, UA. 695 C.N.R.S., Université Paul Sabatier, 118 route de Narbonne, 31062 Toulouse Cédex. France.
}

nouveau ouest-est du cours inférieur. Le lit est étroit (5 à $7 \mathrm{~m}$ ), encaissé, jusqu'à Saint-Lary, avec une pente moyenne supérieure à $2 \%$. Il s'étale au contraire largement ( $25 \mathrm{~m}$ en moyenne) dans la basse vallée, avec une pente moyenne inférieure à $1 \%$ en aval de Sarrancolin.

L'importance des étages subalpin et alpin sur le bassin-versant a pour conséquence un régime des eaux de type nival de transition, avec une crue importante d'avril à juillet, souvent une courte crue d'automne, un étiage d'août-septembre et un autre d'hiver.

La Neste d'Aure et son affluent la Neste du Louron ont fait l'objet d'aménagement nombreux : centrales hydro-électriques au fil de l'eau ou avec des biefs à débits réservés, débits quotidiens constants ou modulés en fonction de la consommation 


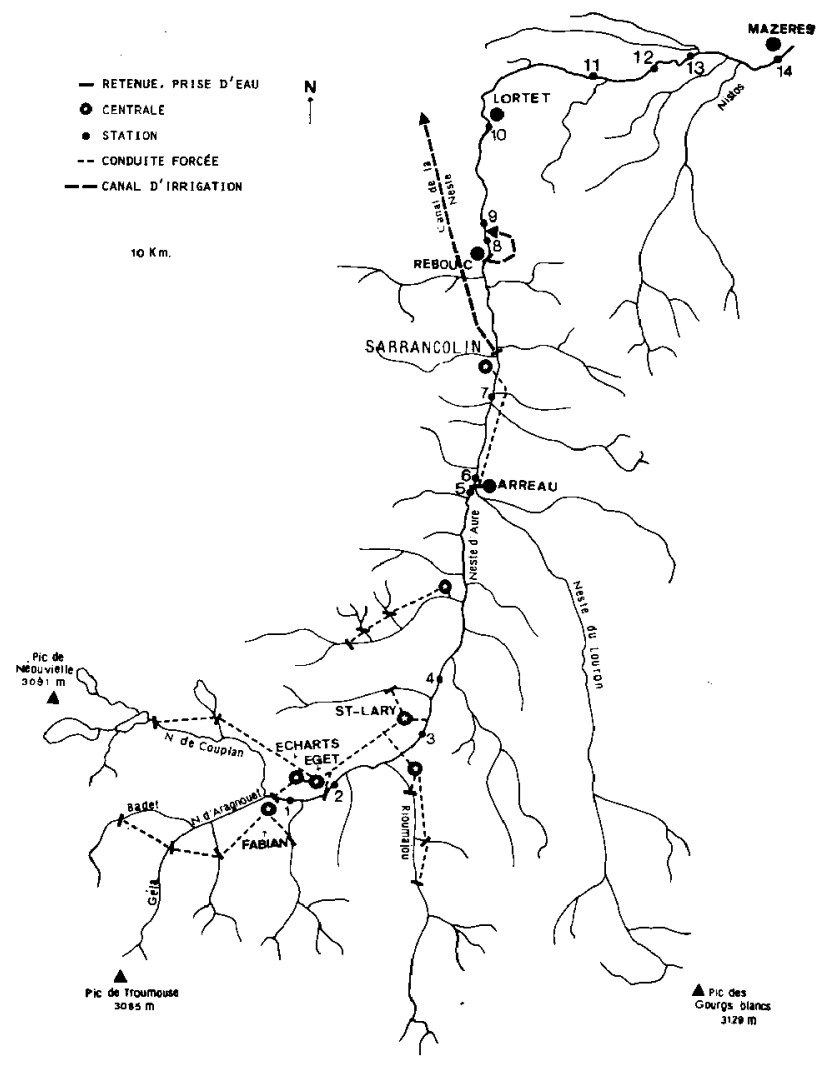

Fig. 1 : Le réseau hydrographique et les aménagements hydro-électriques de la Neste d'Aure 
Tableau I : Les stations et leurs pararnètres, A : altitude. Tm : température moyenne. L : largeur du lit. D : distance à la source

\begin{tabular}{|c|c|c|c|c|c|c|c|}
\hline & stations & $\stackrel{A}{(m)}$ & $\underset{(e ́ t e ́\})}{T \cdot m}$ & $\begin{array}{c}\text { T.m } \\
\text { (hiver) }\end{array}$ & $\begin{array}{l}\text { Pente } \\
\text { (8) }\end{array}$ & $\underset{(m)}{\text { L }}$ & D. \\
\hline 1 & Pont du Moudang & 1060 & 13,1 & 3,6 & 2,66 & 7.5 & 12,8 \\
\hline 2 & Eget (aval retenue) & 980 & 14,1 & 4,7 & 2,1 & 4,5 & 15,1 \\
\hline 3 & $\begin{array}{c}\text { Saint-Lary (carriere } \\
\text { de Mir) }\end{array}$ & 900 & 13,6 & 5 & 3,2 & 6,5 & 19,2 \\
\hline 4 & Vielle-Aure & 790 & 12,4 & 4,4 & 1,21 & 10 & 22 \\
\hline 5 & Arreau "camping" & 700 & 13,8 & 5,5 & 0,98 & 15 & 31,2 \\
\hline 6 & Arreau (aval retenue) & 690 & 14,6 & 4,9 & 1,03 & 10.5 & 32,9 \\
\hline 7 & Camous & 651 & 14,5 & 4,8 & 1,6 & 12.5 & 36,4 \\
\hline 8 & Rebouc (bras réservé) & 600 & 14,8 & 4,6 & 0,59 & 6 & 45 \\
\hline 9 & Rebouc (bras naturel) & 600 & 14,9 & 4,8 & 0,59 & 20 & 45 \\
\hline 10 & Lortet & $\mathbf{5 5 0}$ & 14,2 & 4,8 & 0,92 & 24,5 & 50.8 \\
\hline 11 & Bizous & 490 & 15,6 & 5 & 0.74 & 25 & 60.7 \\
\hline 12 & Anères & 476 & 17 & 5,1 & 0,60 & 25 & 61.5 \\
\hline 13 & Saint Laurent & 463 & 16,9 & 5 & 0,64 & 26 & 63.6 \\
\hline 14 & Mazères & 427 & 16,1 & 6,3 & 0.75 & 26,5 & 64.8 \\
\hline
\end{tabular}

hydro-électrique. Au niveau de Sarrancolin, un canal détoume jusqu'à $12 \mathrm{~m}^{3 /} \mathrm{sec}$ pour l'irrigation des coteaux de Gascogne, ne laissant le plus souvent dans la basse Neste, en été, qu'un débit réservé de $3 \mathrm{~m}^{3 /} \mathrm{sec}$.

\section{Les stations et leurs paramètres (Tableau I).}

Benthos et dérive ont été étudiés dans 14 stations ; celles-ci ont été implantées à la fois en fonction de l'altitude et des différents types d'aménagements. Les prélèvements ont été effectués en juillet, août. octobre 1983 et mars 1984 . Seul le benthos a été prélevé en juillet 1984 .

Sur le tableau I figurent, pour chaque station, l'altitude, les températures moyennes d'hiver et d'été, la pente, la largeur du lit et la distance à la source. La température moyenne estivale s'élève de $1,1^{\circ} \mathrm{C}$ entre les stations 1 et 10 , en $38 \mathrm{~km}$; elle s'élève par contre de plus de $2,5^{\circ} \mathrm{C}$ en $14 \mathrm{~km}$, dans la basse vallée.

\section{Les Simuliidae de la Neste d'Aure}

\subsection{Les espèces récoltées}

26 taxons ont été recensés. Ils sont pour la plupart largement répandus dans la zone paléarctique (Zwick 1978):
Prosimulium * 1 hirtipes (Fries 1824)

(Prosimulium)

2 rufipes (Meigen 1830)

* 3 tomosvaryi (Enderlein 1921)

Simulium

(Eusimulium) 4 latizonum Rubzov 1956

$5 \mathrm{gr}$. aureum

(Nevermannia) 6 armoricanum Doby \& David 1961

7 brevidens (Rubzov 1956)

8 carthusiense Grenier \& Dorier 1958-59

9 cryophilum (Rubzov 1959)

10 gr. vermum

* 11 angustitarse (Lundst röm 1911)

(Obuchovia) 12 auricoma (Meigen 1818)

(Odagmia) * 13 niriditrons Edwards 1920

* 14 ornatum (Meigen 1818)

* 15 spinosum (Doby \& Deblock 1957)

(Simulium) 16 argenteostriatum Strobl 1898 17 reptans (Linné 1758)

18 argyreatum Meigen 1838

19 monticola Friedrichs 1920

* 20 noelleri Friedrichs 1920

* 21 posticatum Meigen 1838

* 22 gaudi Grenier \& Faure 1956

23 variegatum Meigen 1818

* 24 verecundum Stone \& Jamnback 1955

(Tetisimulium) 25 bezzit (Corti 19/6)

(Wilhelmia) *26 equinum (Linné 1758)

Les espèces précédées d'un astérisque ne sont pas citées par Lavandier (1976).

Sur ces 26 taxons, 7 représentent la presque totalité des Simuliidae récoltés et ont seuls un poids 


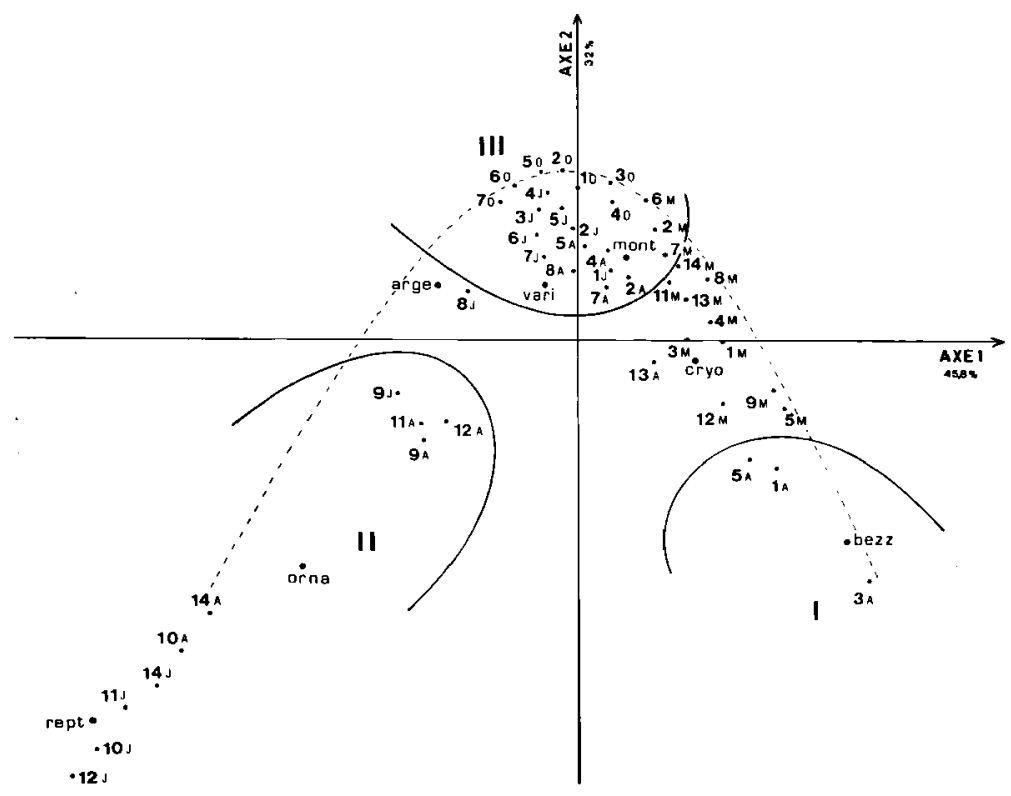

Fig. 2. Analyse factorielle des correspondances stations-espèces sur la dérive.

Trait plein : groupements d'espèces sur les axes I et II. $\mathbf{M}$ : mars. J : juillet. A : août. $\mathbf{O}$ : octobre. Les lettres correspondant aux mois sont précédées des numéros des stations

important dans les analyses statistiques (Tableaux II et III, fig. 2) : S. variegatum, S. bezzii, S. gr. monticola, S. reptans, S. cryophilum, S. ornatum, $S$. argenteostriatum.

S. variegatum, polyvolt in, colonise toutes les stations et en toutes saisons (sauf, pratiquement, en octobre). Dominant en été dans les stations 1 à 10 , il est présent partout en mars, mais avec de faibles effectifs dans le benthos et de façon sporadique dans la dérive. Il représente plus de $50 \%$ des effectifs de la dérive $(13 \%$ dans la haute vallée selon ClergueGazeau \& Gażagnes 1986).

$S$. bezzii, eurytherme, ne dépasse guère $1200 \mathrm{~m}$. Mais il colonise les moyenne ct basse vallées en été (jusqu'à la station 11). Après une disparition quasi totale en octobre, il réapparaît dans la dérive de toutes les stations, en mars, avec de faibles effectifs, et de façon sporadique dans le benthos (st. 5). L'espèce est caractérisée par une dérive importante en août.

S. gr. monticola: deux espèces sont réunies dans ce groupe, $-S$. monticola s. str. et S. argyreatum,- qui se différencient seulement aux stades larve mature et nymphe. Elles ne peuvent donc être séparées dans les analyses statistiques, qui doivent tenir compte de l'ensemble des stades. En été, $S$. argyreatum est un élément normal du benthos jusqu'a la station 10 , tandis que $S$. monticola s. str. est limité aux premières stations. En mars, S. gr. monticola est le seul taxon, avec $S$. variegatum, qui colonise l'ensemble des moyennes et basses vallées, sporadique dans le 
Tableau I1: Données quantitatives sur la dérive (nombre d'individus dérivant pour $1000 \mathrm{~m}^{3}$

\begin{tabular}{|c|c|c|c|c|c|c|c|c|c|c|c|c|c|c|c|c|c|c|c|c|c|}
\hline 罯. & 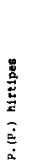 & 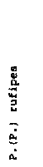 & 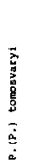 & 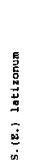 & 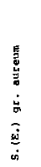 & 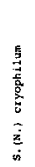 & 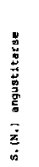 & 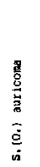 & 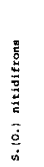 & 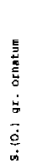 & 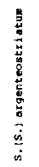 & 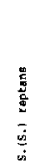 & 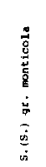 & 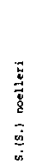 & 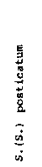 & 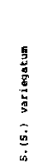 & 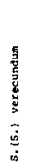 & 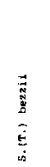 & $\overrightarrow{\tilde{\sigma}}$ & 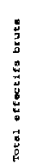 & 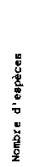 \\
\hline $\begin{array}{r}1 . J \\
\text { J } \\
0 \\
R \\
\end{array}$ & & & & & & $\begin{array}{r}11 \\
70 \\
2\end{array}$ & & 2 & 26 & & 2 & & $\begin{array}{r}57 \\
368 \\
4\end{array}$ & & & $\begin{array}{r}155 \\
1767 \\
3 \\
2\end{array}$ & & $\begin{array}{r}30 \\
2469 \\
2\end{array}$ & $\begin{array}{r}257 \\
4700 \\
3 \\
10\end{array}$ & $\begin{array}{r}136 \\
1072 \\
1 \\
5\end{array}$ & $\begin{array}{l}6 \\
5 \\
1 \\
4\end{array}$ \\
\hline $\begin{array}{l}2 \stackrel{J}{J} \\
\stackrel{2}{\circ} \\
\stackrel{M}{M}\end{array}$ & & & & & & $\begin{array}{r}13 \\
21 \\
8 \\
10\end{array}$ & & & & & & & $\begin{array}{r}30 \\
869 \\
37\end{array}$ & & & $\begin{array}{r}170 \\
2468 \\
11 \\
3\end{array}$ & & $\begin{array}{r}5 \\
555\end{array}$ & $\begin{array}{r}226 \\
3913 \\
19 \\
52\end{array}$ & $\begin{array}{r}89 \\
1651 \\
5 \\
10\end{array}$ & $\begin{array}{l}1 \\
2 \\
3\end{array}$ \\
\hline $\begin{array}{l}3 \mathrm{~J} \\
\mathrm{O} \\
\mathrm{O}\end{array}$ & & & 3 & & & $\begin{array}{l}10 \\
90 \\
9\end{array}$ & & & & $\begin{array}{r}31 \\
3\end{array}$ & & & $\begin{array}{r}33 \\
319 \\
67\end{array}$ & & & $\begin{array}{r}180 \\
995 \\
189 \\
47\end{array}$ & & $\begin{array}{r}3825 \\
37\end{array}$ & $\begin{array}{r}223 \\
5160 \\
189 \\
161\end{array}$ & $\begin{array}{r}0 \\
1827 \\
41 \\
52\end{array}$ & $\begin{array}{l}3 \\
5 \\
1 \\
6\end{array}$ \\
\hline $\begin{array}{r}4 . J \\
n \\
0 \\
n\end{array}$ & & & 11 & & 12 & $\begin{array}{l}16 \\
23\end{array}$ & & & & 39 & 32 & & $\begin{array}{r}45 \\
1934 \\
132\end{array}$ & & & $\begin{array}{r}2543 \\
3083 \\
620 \\
33\end{array}$ & & $\begin{array}{r}54 \\
506 \\
55\end{array}$ & $\begin{array}{r}2690 \\
5599 \\
620 \\
231\end{array}$ & $\begin{array}{r}503 \\
2025 \\
89 \\
21\end{array}$ & $\begin{array}{l}5 \\
6 \\
1 \\
4\end{array}$ \\
\hline $\begin{array}{ll}5 \stackrel{0}{A} \\
\hat{0} \\
\end{array}$ & & a & & & & $\begin{array}{r}73 \\
42 \\
6\end{array}$ & & & & & $\begin{array}{l}30 \\
25\end{array}$ & 20 & $\begin{array}{l}51 \\
25 \\
22\end{array}$ & & & $\begin{array}{l}1390 \\
1014 \\
1307\end{array}$ & 11 & $\begin{array}{r}30 \\
1115 \\
35\end{array}$ & $\begin{array}{r}1522 \\
2252 \\
1313 \\
37\end{array}$ & $\begin{array}{r}359 \\
804 \\
214 \\
5\end{array}$ & $\begin{array}{l}6 \\
7 \\
2 \\
2\end{array}$ \\
\hline $\begin{array}{l}6 \mathrm{~J} \\
\mathrm{a} \\
\mathrm{M}\end{array}$ & 55 & & & & 44 & $\begin{array}{l}44 \\
13 \\
16\end{array}$ & & & & 35 & 26 & 17 & $\begin{array}{r}61 \\
119 \\
21\end{array}$ & & & $\begin{array}{l}1395 \\
1624 \\
1276\end{array}$ & & 186 & $\begin{array}{r}1570 \\
2303 \\
1292 \\
76\end{array}$ & $\begin{array}{r}179 \\
535 \\
248 \\
11\end{array}$ & $\begin{array}{l}6 \\
6 \\
2 \\
2\end{array}$ \\
\hline $\begin{array}{ll}7 & J \\
A \\
g \\
M\end{array}$ & & & & & & $\begin{array}{l}27 \\
27 \\
31 \\
31\end{array}$ & & & & 5 & 22 & $\begin{array}{r}127 \\
40\end{array}$ & $\begin{array}{r}280 \\
486 \\
3 \\
135\end{array}$ & & & $\begin{array}{r}3022 \\
1721 \\
38 \\
88\end{array}$ & & $\begin{array}{r}220 \\
347 \\
27\end{array}$ & $\begin{array}{r}3697 \\
2621 \\
44 \\
275\end{array}$ & $\begin{array}{r}673 \\
592 \\
12 \\
53\end{array}$ & $\begin{array}{l}7 \\
5 \\
3 \\
4\end{array}$ \\
\hline $\begin{array}{r}8 \stackrel{J}{A} \\
0 \\
M \\
\end{array}$ & 9 & & & 20 & & 8 & 82 & 3 & & $\begin{array}{r}11 \\
3\end{array}$ & 171 & 46 & $\begin{array}{r}308 \\
133 \\
36\end{array}$ & & 3 & $\begin{array}{l}3110 \\
237\end{array}$ & & $\begin{array}{r}20 \\
29 \\
4\end{array}$ & $\begin{array}{r}4170 \\
435 \\
0 \\
57\end{array}$ & $\begin{array}{r}612 \\
166 \\
0 \\
13\end{array}$ & $\begin{array}{l}8 \\
9 \\
0 \\
4\end{array}$ \\
\hline $\begin{array}{l}9 \\
9 \\
\text { A } \\
\text { : } \\
:\end{array}$ & $s$ & & & & & 6 & & & & $\begin{array}{r}74 \\
2\end{array}$ & 88 & $\begin{array}{r}489 \\
68\end{array}$ & $\begin{array}{r}230 \\
34 \\
24\end{array}$ & & & $\begin{array}{r}1422 \\
173\end{array}$ & & $\begin{array}{l}22 \\
28 \\
15\end{array}$ & $\begin{array}{r}2325 \\
311 \\
0 \\
14\end{array}$ & $\begin{array}{c}319 \\
145 \\
0 \\
9\end{array}$ & $\begin{array}{l}6 \\
6 \\
0 \\
3\end{array}$ \\
\hline $\begin{array}{r}10 \stackrel{J}{\mathrm{~A}} \\
\mathrm{~A} \\
\mathrm{~B} \\
\mathrm{H}\end{array}$ & & & & & e & B & & & & 13 & & $\begin{array}{l}197 \\
459\end{array}$ & 32 & & & 327 & & a & $\begin{array}{r}286 \\
855 \\
0 \\
0\end{array}$ & $\begin{array}{r}70 \\
168 \\
0 \\
0\end{array}$ & $\begin{array}{l}2 \\
7 \\
0 \\
0\end{array}$ \\
\hline $11 \stackrel{\mathrm{J}}{\mathrm{A}}$ & & & & & & & & & & & & $\begin{array}{r}793 \\
24\end{array}$ & $\begin{array}{l}30 \\
99\end{array}$ & 3 & & $\begin{array}{r}480 \\
36 \\
37\end{array}$ & & $\begin{array}{c}7 \\
25\end{array}$ & $\begin{array}{r}1273 \\
96 \\
0 \\
161\end{array}$ & $\begin{array}{r}252 \\
32 \\
0 \\
13\end{array}$ & $\begin{array}{l}2 \\
5 \\
0 \\
3\end{array}$ \\
\hline $\begin{array}{r}12 \mathrm{~J} \\
\mathrm{~A} \\
0 \\
\mathrm{M}\end{array}$ & & & & & & & & & & 64 & 15 & $\begin{array}{c}383 \\
51\end{array}$ & 97 & & & $\begin{array}{r}149 \\
95 \\
28\end{array}$ & & $\begin{array}{l}15 \\
23\end{array}$ & $\begin{array}{r}596 \\
213 \\
0 \\
57\end{array}$ & $\begin{array}{c}55 \\
29 \\
0 \\
10\end{array}$ & $\begin{array}{l}3 \\
5 \\
0 \\
3\end{array}$ \\
\hline 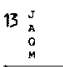 & & & & & & & & & & 77 & & $\begin{array}{r}28 \\
4\end{array}$ & $\begin{array}{r}100 \\
20\end{array}$ & & & $\begin{array}{r}714 \\
21 \\
5\end{array}$ & 14 & $\begin{array}{r}328 \\
6\end{array}$ & $\begin{array}{r}77 \\
1184 \\
29 \\
34\end{array}$ & $\begin{array}{l}13 \\
13 \\
25 \\
22\end{array}$ & $\begin{array}{l}1 \\
5 \\
2 \\
3\end{array}$ \\
\hline $\begin{array}{r}14 \mathrm{~J} \\
8 \\
\mathrm{O} \\
\end{array}$ & & & & & & & & & & 27 & & $\begin{array}{l}42 \\
42\end{array}$ & 23 & & & $\begin{array}{l}30 \\
40 \\
4\end{array}$ & & $\begin{array}{l}2 \\
4\end{array}$ & $\begin{array}{c}99 \\
94 \\
0 \\
31\end{array}$ & $\begin{array}{r}10 \\
38 \\
0 \\
6\end{array}$ & $\begin{array}{l}3 \\
3 \\
0 \\
3\end{array}$ \\
\hline TOTAL & 54 & 8 & 14 & 23 & 64 & 512 & 82 & 5 & 26 & 410 & 411 & 3216 & 6505 & 3 & 3 & 32059 & 25 & 10004 & 53472 & & 18 \\
\hline $\begin{array}{l}\text { Total } \\
\text { erfect if } \\
\text { bruts }\end{array}$ & ${ }_{10} 10$ & 2 & 2 & 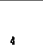 & 10 & 168 & 12 & 2 & 6 & 92 & 71 & 759 & 1905 & 1 & 1 & 7078 & 5 & 3032 & & 1376 & \\
\hline$:$ & 0.07 & 0.01 & 0,01 & 0,03 & 0,07 & 1 & 0,09 & 0,01 & 0,04 & 0.7 & 0.5 & 5,5 & 13,9 & 0,007 & 0,007 & 55,9 & 0,04 & 22 & & & \\
\hline
\end{tabular}


Tableau III : Données quantitatives sur les prélèvements benthiques. Effectifs bruts (courants lent et rapide confondus)

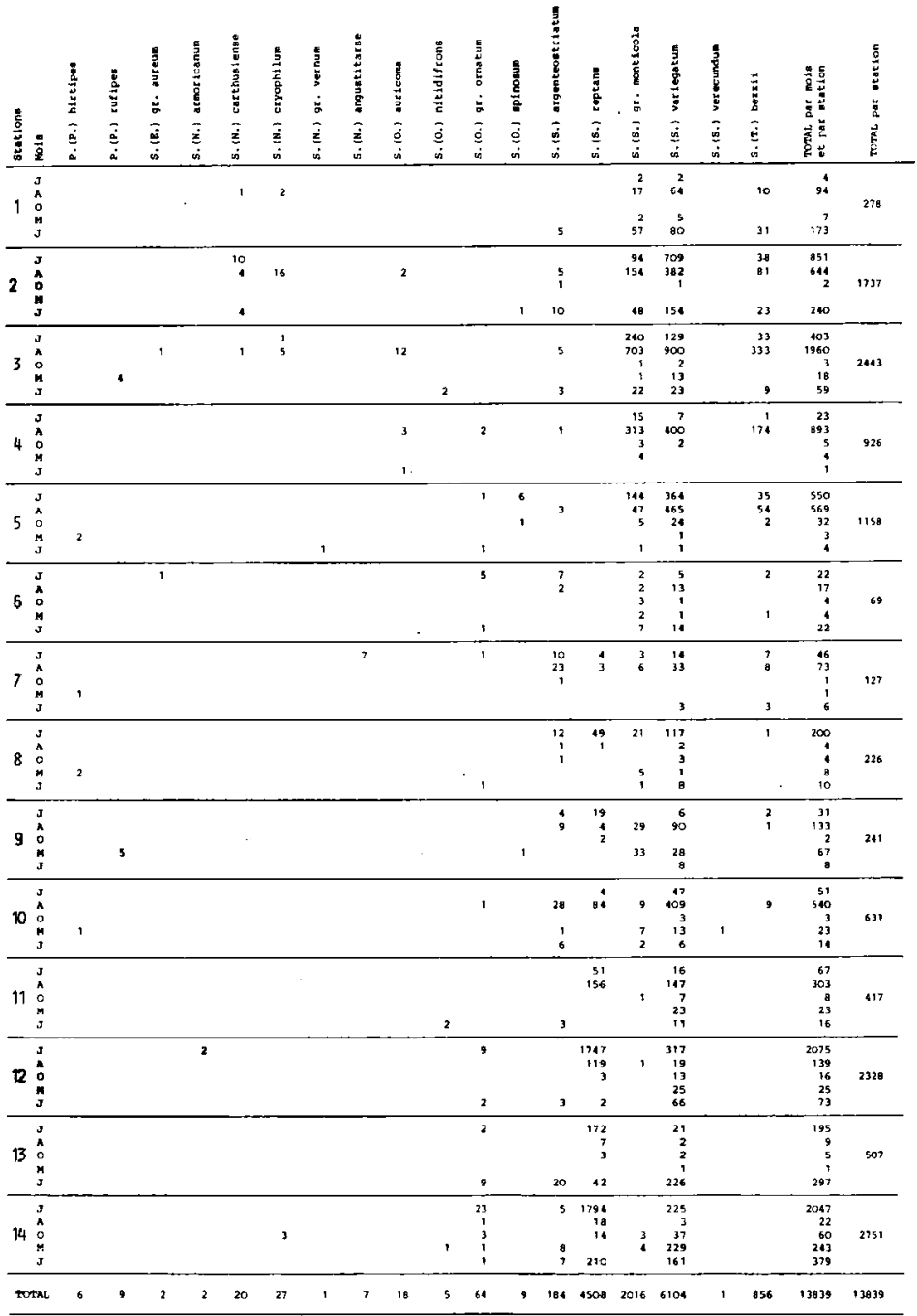


benthos des stations 11 à 14 , mais régulier dans la dérive de toutes les stations.

S. reptans, univoltin, colonise surtout la basse vallée et constitue l'essentiel du benthos en aval de Lor. tet (st. 11 à 14) durant l'été. Il est sporadique en amont de la station 6 .

S. cryophilum est une espèce de moyenne et haute altitude (jusqu'à $2500 \mathrm{~m}$ ), qui colonise les stations 1 à 3 en juillet 1983 et figure ensuite dans la dérive jusqu'à Lortet (st. 10). Elle disparaît en octobre 1983, est rare en mars 1984 et n'a pas été retrouvée en juil. let 1984

S. ornatum, polyvoltin. Espèce eurytherme, dans le benthos et la dérive des stations 3 à 14 en juillet et août 1983, sporadique en octobre et mars. En juillet 1984, elle n'a plus été recueillie qu'à Eget (st. 2, $0,42 \%$ du benthos).

S. argenteostriattsm, univolt in, est rare $(0,74$ à $4 \%)$ dans le benthos et la dérive des stations 4 à 9 en juillet 1983. Absent en août, octobre et mars, il réapparaît en juillet 1984, à la fois dans la basse vallée (st. 10 à 14) et les stations 1 et 2 (à débit réservé faible).

La présence des autres taxons est sporadique, très localisée et toujours avec de faibles effectifs. Ces taxons peuvent être répartis en trois groupes:

- les uns, - comme les especes du genre Prosimulium, S. carthusiense, ..., atteignent dans la moyenne vallée de la Neste leur limite inférieure de répartition. En été, $S$. carthusiense ne descend pas audessous de la station $3 ; \operatorname{Pr}$. hirtipes atteint la station 10 , mais en mars.

- certains,- comme $S$. posticatum et $S$. equinum, sont caractéristiques des grandes rivieres de plaine ; ils colonisent quelques stations de la basse Neste, avec de très faibles effectifs.

- d'autres,-comme S. verecundum et S. noelleri;auraient leur habitat dans la Neste mème, mais très localisé.

Enfin, S. gaudi colonise un affluent de la Neste, l'Esponne, coulant sur des affleurements calcaires. Larves et exuvies nymphales sont entraînées dans la Neste par la dérive.

Les premières recherches sur les Simuliidae de la vallée d'Aure (Lavandier 1976) ont porté surtout sur la haute vallée, de sorte que les espèces non encore signalées sont essentiellement des formes de basse et moyenne altitude. Sept d'entre elless ne dépassent pas $700 \mathrm{~m}$ (fig. 3). Les espèces qui donnent l'impression, par leur capture, d'être présentes à toutes altitudes sont en fait sporadiques au-dessous de $800 \mathrm{~m}$ (S. cryophilum, par exemple) ou au-dessus de $1200 \mathrm{~m}$ (S. variegatum). S. gr. monticola est fina. lement le seul taxon figurant régulièrement dans la dérive et le benthos à toutes altitudes.

\subsection{Relations entre les peuplements de la Neste d'Aure, des Pyrénées occidentales et orientales.}

Les étages alpin et subalpin occupent une vaste surface sur les bassins versants des Pyrénées centrales. Leur importance diminue vers les Pyrénées occidentales et orientales, et par conséquent la durée de l'enneigement et de la crue printanière. Vinçon (1987) a observé dans la vallée d'Ossau la présence d'espèces comme $S$. variegatum, $S$. bezzii,..., à des altitudes plus élevées que dans la vallée d'Aure. De même, Grenier (1949) et Jarry (1975) signalent $S$. bezzii à l'altitude de $2000 \mathrm{~m}$ dans le déversoir du lac des Bouillouses (Pyrénées-orientales)

Sur les rivières à régime pluvio-nival dominant des Pyrénées-atlantiques, certaines espèces de la basse Neste sont rares ou absentes. Par contre apparaissent des espèces non signalées dans les Pyrénées centrales, comme $S$. carthusiense f. truncata, S. lineatum ou $S$. hispaniola. Cette demière espèce colonise le Saison, rivière de Piérnont, sur une longue distance.

\subsection{Le benthos de juillet, en 1983 et 1984 .}

15 taxons ont été recueillis dans le benthos de juil let, au total; 14 en 1983 et 11 en 1984 . On constate en 1984 la disparition de 3 espèces rares en 1983 : $S$. latizonum, $S$. angustitarse et $S$. armoricanum (benthos d'Arreau).

S. cryophilum disparaît en 1984, mais les autres espèces dominantes sont présentes. Deux d'entre elles, $S$. variegatum et $S$. gr. monticola conservent la même répartition,- respectivement des stations 1 à 14 et 1 à 9 . S. reptans parvenait jusqu'à la station 6 en juillet 1983 ; il ne colonise plus la Neste que des stations 1 I à 14 en juillet 1984 .

Le fond du peuplement paraît identique en 1983 et 1984, mais deux espèces ont une aire de répartition plus restreinte,- $S$. cryophilum vers la haute vallée et $S$. reptans vers la basse vallée. En novembre 1982, une crue violente avait bouleversé le lit de la Neste d'Aure au-dessous de $1300 \mathrm{~m}$. S. cryophilum 


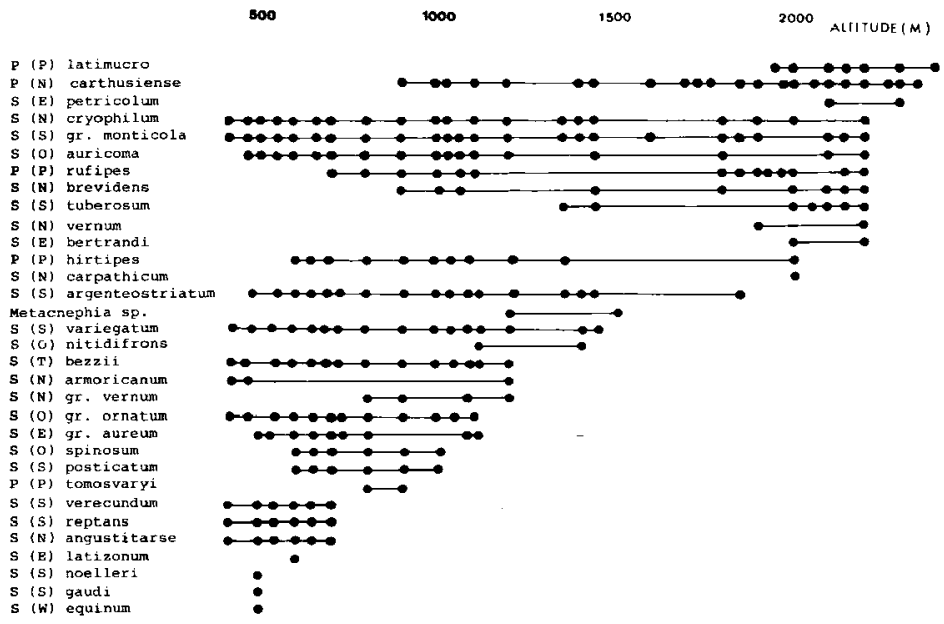

Fig. 3 : Répartition altitudinale des Simulidae

et $S$. reptans ont peut-être étendu leur domaine durant la phase de recolonisation. Quant aux espèces qui ont disparu, il s'agit probablement d'un phénomène analogue à celui observé par Angelier et al. (1985) à propos des Hydrachnellae : à côté des espèces dominantes, on trouve dans chaque station des espèces apportées par la dérive, et dont la présence est aléatoire.

\section{Ecologie des espèces dominantes}

Les tableaux II et III (données de la dérive et du benthos) permettent de suivre grossièrement le cycle des espèces et aussi les successions amont-aval. C'est en juillet-août que les effectifs de Simuliidae sont. globalement, les plus importants :

\begin{tabular}{|c|c|c|c|c|}
\hline & juillet & août & octobre & mars \\
\hline $\begin{array}{l}\text { benthos } \\
\text { (environ } 1.6 \mathrm{~m}^{2} \text { ) } \\
\text { dérive }\end{array}$ & 6565 & 5400 & 140 & 394 \\
\hline (ind./1000 $\mathrm{m}^{3}$ ) & 18011 & 30480 & 2509 & 951 \\
\hline
\end{tabular}

Deux types d'analyses multivariables ont été réalisées : une analyse factorielle des correspondances sur la dérive des 7 espèces dominantes et une classification par la méthode des nuées dynamiques. La première est basée sur les effectifs dérivant dans $1000 \mathrm{~m}^{3} \mathrm{~d}$ 'eau ; la seconde prend en compte à la fois les données de la dérive et celles du benthos en courant lent et rapide. 


\subsection{Interprétation de l'A.F.C. sur la dérive (fig-3)}

Trois axes rendent compte de $91,1 \%$ de l'inertie du nuage des points.

- L'axe I $(45,8 \%$ de l'inertie) oppose S. reptans et $S$. ornatum, ainsi que les stations 10 à 14 en juilletaoût, à $S$. bezzii et aux stations les plus élevées, en août. $S$. variegatum et $S$. gr. monticola se situent près de l'origine de l'axe. Celui-ci représente un facteur amont-aval classique, traduisant la réduction de la pente, l'accroissement de la température..., et la succession des espèces dans la dérive qui en est la conséquence.

- L'axe II ( $32 \%$ de l'inertie) oppose les stations et espèces précédentes, à $S$. variegatum et $S$. gr. monticola et à un groupe de stations : 1 à 8 de juillet à octobre (sauf 1, 3 et 5 en août) et secondairement en mars. C'est en fait l'opposition entre les espèces à large répartition (positives sur l'axe) et des espèces plus spécialisées.

Le plan engendré par les axes I et II permet de différencier 3 types de dérive selon les stations et la période de l'année,- caractérisées respectivement par $S$. bezzii, $S$. reptans - S. omatum, et $S$. variegatum-S. gr, monticola.

- L'axe III (13\% de l'inertie) isole $S$. gr. monticola et l'ensemble des stations en mars, et les oppose aux autres espèces et dérives de juillet, août et octobre. Le gr. monticola est le taxon dominant en mars. Selon Dorier (1962), les deux espèces qui le constituent sont, parmi les especes fondamentales, celles dont la nymphose est la plus précoce.

\subsection{Interprétation de la classification par la méthode des nuées dynamiques (Tableau IV)}

La matrice des données comportait, outre les relevés des dérives, ceux du benthos en courant lent et rapide et 62 variables taxinomiques (de 8 à 16 espèces pour chacune des périodes de prélèvement).

La partition en 5 classes retenues après 15 tirages explique $85,2 \%$ de l'inertie des données. Les classes de partition sont interprétées par la structure du peuplement et les paramètres altitude, pente, température minimale et maximale de l'eau, degrés-jours annuels où la température dépasse 5 , 10 et $15^{\circ} \mathrm{C}$.

- Classes 1 et 2 : elles correspondent aux dérives au-delà de $800 \mathrm{~m}$ (stations $\mathrm{I}$ à 4) avec $S$. bezzii, $S$. variegatum et $S$. gr. monticola, -accessoirement $S$. cryophilum. La pente est élevée et le nombre de
Tableau IV : Nuées dynamiques. Classes de la partition du meilleur critère. 1 à 14 : stations. $L$ : courant lent. $R$ : courant rapide. $D$ : dérive.

Les termes lent et rapide désignent dans chaque station deux types de courant ; ils n'ont pas de valeur comparative rigoureuse d'une station à l'autre.

\begin{tabular}{|c|c|c|c|c|c|c|c|c|c|c|c|c|c|c|c|}
\hline & & 1 & 2 & 3 & 4 & 5 & 6 & 7 & B & 9 & 10 & 11 & 12 & 13 & 14 \\
\hline Classe & 1 & D & & $D$ & & & & & & & & & & & \\
\hline Classe & 2 & & D & & D & & & & & & & & & & \\
\hline Classe & 3 & & $\mathbf{R}$ & $\begin{array}{l}\mathbf{L} \\
\mathbf{R}\end{array}$ & $\mathbf{L}$ & $\begin{array}{l}\mathbf{R} \\
\mathbf{D}\end{array}$ & D & D & & D & R & & & & \\
\hline Clasge & 4 & $\begin{array}{l}\mathbf{L} \\
\mathbf{R}\end{array}$ & $\mathbf{L}$ & & $\mathbf{R}$ & $\mathrm{L}$ & $\begin{array}{l}\mathrm{L} \\
\mathbf{R}\end{array}$ & $\begin{array}{l}\mathrm{L} \\
\mathbf{R}\end{array}$ & $\begin{array}{l}\mathbf{L} \\
\mathbf{R} \\
\mathbf{D}\end{array}$ & $\begin{array}{l}\mathbf{L} \\
\mathbf{R}\end{array}$ & $\begin{array}{l}\text { L } \\
\text { D }\end{array}$ & $\begin{array}{l}\text { L } \\
\text { R } \\
\text { D }\end{array}$ & $\begin{array}{l}\text { L } \\
\text { D }\end{array}$ & $\begin{array}{l}\mathrm{L} \\
\mathrm{R} \\
\mathrm{D}\end{array}$ & $\begin{array}{l}\text { L } \\
\text { D }\end{array}$ \\
\hline Classe & 5 & & & & & & & & & & & & R & & $\mathbf{R}$ \\
\hline
\end{tabular}

degrés-jours supérieurs à 5 et $10^{\circ} \mathrm{C}$ faible. S. variegatum et $S$. gr. monticola sont prédominants dans les dérives de juillet et celles des st. 2 et 4 en août (classe 1), tandis que $S$. bezzii dérive beaucoup plus dans les stations 1 et 3 en aoùt (classe 2), - les larves matures, nymphes et exuvies nymphales étant alors en nombre relativement élevé.

- Classe 3 : elle correspond au benthos des courants lents et rapides des stations les plus élevées (2 à 5) et de la station 10 (Lortet), et aux dérives des stations 5 à 9 . On retrouve le groupement à $S$. variegatum, $S$. gr. monticola et $S$. bezzii, mais à un niveau plus bas dans la dérive que dans le benthos. A la station 10, le prélèvement en courant rapide a été effectué en un point où le lit était rétréci, avec une rupture de pente sur quelques dizaines de mètres. La dominance de $S$. variegatum, réapparaît avec des effectifs comparables à ceux des stations 2 à 5 . Les effectifs des taxons dominants sont plus élevés que leur moyenne dans l'ensemble des stations.

- CLASSE 4 : elle regroupe 12 des 14 stations du benthos, en courant lent ( 1 et 2,5 à 14), 8 en courant rapide $(1,4,6$ à 9,11 et 13$)$ et les dérives de la basse vallée. Les stations sont caractérisées par un faible débit estival ou bien des biefs à débit réservé. Le caractère commun à l'ensemble des stations de cette classe est la faiblesse des effectifs (effectifs des taxons dominants inférieur à leur moyenne dans l'ensemble des stations).

- Classe 5 : cette classe regroupe le benthos des stations 12 et 14 en courant rapide (lit de la Neste 
rétréci et rupture de pente). Des effectifs élevés (10 fois supérieurs à ceux des st. 11 et 13 en juillet), $S$. reptans dominant en été, $S$. variegatum en hiver. caractérisent cette classe.

\subsection{Les associations de Simuliidae de la moyenne et basse Neste d'Aure.}

A partir des données brutes et des analyses multivariables, il est possible de reconnaître deux associations :

\section{ASSOCIATION 1.}

Elle est constituée d'espèces d'altitudes moyennes, colonisant des milieux à forte pente (supérieure à $2 \%$ ) et basses températures estivales.

Espèces caractéristiques : $S$. bezzii, $S$. cryophilum, $S$. variegatum, S. gr. monticola.

Les effectifs de S. variegatum et $S$. gr. monticola sont nettement plus élevés dans le benthos que ceux de $S$. bezzii et surtout $S$. cryophilum. L'importance de $S$. bezzii dans la dérive des stations 1,3 et 5 en août paraît coïncider avec la nymphose de cette espèce dans des stations où les perturbations quotidiennes du débit sont atténuées.

Au-delà de $1100 \mathrm{~m}, \mathrm{~S}$. variegatum et $\mathrm{S}$. monticola persistent dans les biefs à débits réservés.

Association 2.

Elle caractérise la basse vallée, à faible débit et températures élevées en été, pente faible.

Espèces caractéristiques: $S$. reptans (été), $S$. variegatum.

Espèces accessoires: $S$. ornatum, $S$. argenteostriatum.

Entre les deux associations, une zone de transition, le long de laquelle les espèces de l'association 1 disparaissent progressivement tandis que S. reptans devient l'espèce dominante : ses effectifs croissent progressivement à partir de la station 7 , sur les pentes faibles. L'analyse par la méthode des nuées dynamiques rend compte en partie de cette transition. (classes 3 et 4 ).

\subsection{Associations du benthos et dérive des Simuliidae.}

La classe 1 de la classification par les nuées dynamiques ne regroupe pas benthos et dérives des stations 1 à 5 . Les dérives à $S$. variegatum et $S$. gr. monticula sont proches de celles observees par
Clergue-Gazeau \& Gazagnes (1986) entre 1100 et 1300 $\mathrm{m}$, - bien que le groupe monticola ne soit plus dominant. La classe 3, par contre, regroupe le benthos des stations 2 à 5 et les dérives des stations 5 à 7 et 9 . Il existe par conséquent un décalage vers l'aval des dérives par rapport au benthos, de l'ordre de $14 \mathrm{~km}$ (st. 5 à 9). S. cryophilum, qui ne dépasse pas la station 3 dans le benthos, se retrouve dans la dérive à $25 \mathrm{~km}$ en aval. La Neste du Louron (dont le confluent avec la Neste d'Aure, à Arreau, est situé entre les stations 5 et 6) représente un apport de dérive très important; mais la distance moyenne parcourue par les Simuliidae reste du même ordre, quelle que soit l'origine de l'apport. D'autre part, les petits affluents de la Neste, entre les stations 5 et 9 , n'ont pas un débit tel qu'ils puissent imprimer leur cachet à la Neste par leur apport de dérive. $S$. gaudi, par exemple, qui dérive dans la Neste à partir du ruisseau d'Esponne, est sans influence sur le cours d'eau axial. C'est à partir de Rebouc (st. 8 et 9) que la dérive rend compte de la présence de $S$. reptans dans le benthos.

La dérive rend par conséquent compte globalement des associations de Simuliidae d'une rivière. Mais le décalage amont-aval entre benthos et dérive est trop important pour permettre de suivre la succession des especes par la dérive.

\subsection{Impact des aménagements sur la faune simulidienne.}

Les aménagements hydro-électriques ont pour conséquence, jusqu'à Rebouc (st. 9) la présence de biefs à débit réservé constant ou modulé. Le détournement des eaux vers le canal de la Neste, à Sarrancolin, ne laissant qu'un débit de $3 \mathrm{~m}^{3} / \mathrm{sec}$ dans une rivière au lit très large, il en résulte un ralentissement du courant en été et un réchauffement de l'eau surtout sensible en aval de Lortet (cf. $\S 2$ et Tableau I).

La classe 4 (nuées dynamiques) réunit des stations qui ont en commun la faiblesse des effectifs de Simuliidae et un courant réduit, (1, 2, 4, 5 en partie, 6 à 11,13 ). Ce sont les stations des biefs à débit perturbé et les stations de la basse vallée. Ces caractères opposent la classe 4 à la classe 3 (effectifs de chaque taxon supérieur à sa moyenne sur la Neste).

La réduction du débit,- donc du courant pour une pente et une largeur de lit déterminée,- dans les biefs 
aménagés et la basse vallée a pour conséquence la réduction des effectifs de Simuliidae. Le benthos rend compte immédiatement du phénomène, tandis que la dérive présente un retard dans son expression (cf. $\S 4.4$.).

\subsection{Simuliidae et zonation des cours d'eau}

Zwick (1974), à propos des Simulizdae de la Fulda (sources à l'altitude moyenne de $850 \mathrm{~m}$ ) différencie 2 zones dans le rhithral,- épi- et hyporhithral. Jedlika (1984) reconnait dans le rhithral de la rivière Bora (Carpathes) 3 zones,- épi-, méta- et hyporhithral. L'épirhithral correspond, par les espèces citées, à l'association 1 reconnue sur la Neste (jusqu'à $1200 \mathrm{~m}$ ) et l'hyporhithral à l'association 2 (à $S$. reptans). Le métarhithral correspond à la zone de transition sur la Neste.

Selon Malicky (1978), cette zonation doit être considérée comme un phénomène statistique, la typologie des eaux courantes étant hautement diversifiée d'une section de cours d'eau à l'autre (zones d'ombres, resserrement de la vallée, apports de résurgences froides...), « ces influences étant plus importantes encore dans les sections à faible vitesse du courant ". Ces vues de Malicky sont confirmées sur la Neste d'Aure. Chacune des 2 associations reconnues peut être considérée comme une moyenne, le sommet d'une courbe de Gauss. Le métarhithral de Jedlika n'est en fait que la zone de chevauchement de 2 courbes de Gauss, et non une association véritable.

\section{Conclusions}

32 taxons ont été recensés sur la Neste d'Aure, de $2500 \mathrm{~m}$ à son confluent avec la Garonne $(427 \mathrm{~m})$, dont 26 au-dessous de $1100 \mathrm{~m}$.

Sur ces 26 taxons, 7 sont dominants. La présence des 19 autres est sporadique. Deux associations sont mises en évidence. L'une à $S$. bezzii, S. cryophilum, $S$. variegatum et $S$. gr. monticola; l'autre à $S$. reptans, $S$. variegatum et $S$. ornatum. Elles correspondent à l'épi- et hyporhithral de Jedlicka.
Un décalage amont-aval apparaît entre la composition du benthos et celle de la dérive, celle-ci rendant compte avec retard de la succession des espèces.

L'impact des aménagements hydro-électriques et des prélèvements pour irrigation se traduit par une réduction des effectifs des Simulitiae qui correspond à une réduction du courant. La classification par la méthode des nuées dynamiques regroupe dans une même classe (la classe 4) des stations qui sont caractérisées par des associa tions différentes, au moins en partie, mais qui ont en commun des débits faibles en été et des effectifs de Simulitae réduits.

\section{Travaux cites}

Angelier (E.), Angelier (M.L.) \& Lauga (3.). 1985. - Recherches sur l'écologie des Hydracariens (Hydrachnellae, Acari) dans les eaux courantes. Annls Limnol., 21 (1): 25-64.

Clergue-Gazeau (M.) \& Gazagnes (G.) 1986. - Les Simulidae de la Neste d'Aure. (Pyrénés centrales). I. Impact des amenagements hydrólectriques dans la haute vallée. (Diptera, Nematocera). Annls Limnol, 22 (2) : 195-203.

Dorier (A.). 1962-1963. - Documents pour servir à la connaissance des Simuliidae du Sud-Est de la France. Travaux lab. Hydro biol. Piscic. Grenoble, 54-56:7-79.

Grenier (P.) 1949. - Remarques à propos de Simulium bezzii Corti 1916, espèce méditerranéenne présente en France (Dipt. Simuliidae). L'entomologiste, 5 (5-6): 137-140.

Jarry (D.M.). 1975. - Contribution à l'étude des Simulies du Languedoc-Roussillon. III. A propos de six especes nouvelles pour les Pyrénées orientales. Vie et Milieu, 25 (2) : 249-258.

Jedlicka (L.). 1984 - Simuliidengemeinschaften des Flusses Belà und seiner Nebenflüsse. Práce Labor. Rybàar. Hydrobiol., 4 : 193-211.

Lavandier (P.7. 1976. - Premières données sur la répartition des Simuliidae (Diptères, Nématocères) dans la vallée d'Aure (Hautes-Pyrénées, France). Bull. Soc. Hist. nat. Toulouse, 112 (1-2) : 89-95

Malicky (H). 1978. - Der Temperaturgang einiger niederösterreichischer Gebirgsbäche. Wetter und Leben., 30: 170-183.

Vinçon (G.). 1987. - Comparaison de la faume benthique des val. lées d'Aure et d'Ossau, en vue de l'élaboration d'tune méthodo logie de surveillance des cours d'eau de montagne. These de Docteur Ingénieur. Spécialité : Ecologie, Toulouse, 381 p.

Zwick (H.). 1974. - Faunistisch-ökologische und taxonomische Untersuchungen an Simuliidae (Diptera) unter besonderer Beruicksichtigung der Arten des Fulda-Gebietes. Abbandl. Senckenberg. Naturf. Gesellschaft, $533: 1-116$.

Zwick (H.). 1978. - Simuliidae. In Illies (J.) : Limnofauna Euro paea. G. Fischer Verlag, Stuttgart : $404-440$. 